\title{
Clinical and Histopathological Correlation in Hansen's Disease
}

\author{
Muruganantham Arunagirinathan, Vennila Muniswamy* and J. Sivaraman \\ Dept of Pathology, Govt. Vellore Medical College \& Hospital, Tamil Nadu, India
}

\begin{abstract}
Background: The present study was carried out to correlate clinical diagnosis of new leprosy cases with that of histopathological diagnosis of skin biopsies stained with Haematoxylin and Eosin followed by Fite Faraco stain.

Method: Skin biopsies of 70 newly diagnosed leprosy patients were fixed in $10 \%$ formalin and routine histopathological examination done followed by special stain (Fite Faraco) to evaluate the bacterial index.

Results: From this study it was observed that, the commonest age group affected by leprosy was 31 to 50 years, males are twice more commonly affected than females $(\mathrm{M}: \mathrm{F}=1.9: 1)$ and the most commonest clinically diagnosed spectrum was Tuberculoid leprosy (TT). The commonest histopathologically diagnosed spectrum was Indeterminate leprosy(IL). It was observed that there was complete agreement between clinical diagnosis and histopathological diagnosis in $62.85 \%$ cases and disagreement was observed in $37.15 \%$ cases.

Conclusion: In case of confirmed discrepancy, the more advanced findings (ie. towards the lepromatous pole) should be given greater weightage and the case is to be classified and treated accordingly.
\end{abstract}

Keywords: Clinical Diagnosis, Histopathological Diagnosis, Leprosy, Fite Faraco Stain, Agreement, Disagreement

\section{Introduction}

Leprosy (Hansen's disease), is a chronic infectious disease that primarily affects the skin \& the peripheral nerves. Leprosy is one of the oldest diseases of mankind ${ }^{[1]}$. Even though tremendous progress has been made in the field of leprosy, it still continues to be a global health problem.

Despite an extensive global drug programme for leprosy, implemented by the WHO, leprosy is endemic in many countries with approximately 211,000 new cases reported every year.

The overall prevalence of leprosy in India has declined from $5.27 / 10000$ in the year 2000 to $0.66 / 10000$ in the year 2016, but still it continues to be a sizable public health problem $^{[2]}$. India represents approximately $60 \%$ of the global burden ${ }^{[3]}$.

Leprosy expresses itself in different clinico- pathological forms depending on the immune status of the host. Diagnosis of leprosy is based on different clinical parameters which involves detailed examination of skin lesions and peripheral nerves. Demonstration of acid- fast bacilli in slit skin smears by modified Ziehl- Neelsen's staining also aids in the diagnosis of leprosy. A reliable diagnosis hinges around a good histopathological work up and demonstration of bacilli in histopathological sections.
Fite - Faraco staining procedure has proved most valuable in demonstrating lepra bacilli in tissue sections.

Ridley and Jopling were the first to suggest a subclassification of leprosy based on immunological aspects, as five types; Tuberculoid (TT), Borderline Tuberculoid (BT), Mid borderline (BB), Borderline Lepromatous (BL) and Lepromatous Leprosy $(\mathrm{LL})^{[4]}$. Later, they correlated clinical and bacteriological findings in each group with respective immunological and histological findings.

\section{Materials and Methods}

The study was conducted on the skin biopsies of patients newly registered at the Department of Dermatology, Venerology \& Leprosy and subsequently reported to the Histopathology section of the Department of Pathology, Government Vellore Medical College \& Hospital, Vellore, over a period of 2 years from January 2015 to Dec 2016. Newly diagnosed leprosy patients aged between 15 to 65 years with hypopigmented patches with loss of sensation were included in the study. Patients younger than 15 years and older than 65 years, old treated cases and those who where on multi drug therapy(MDT) were excluded from the study.

After informed consent, enrolled patients were subjected to general and dermatological examination regarding 
the morphology, number, size, site, colour, anaesthesia, margins, surface, satellite lesion and central clearing of skin lesions as well as the involvement of peripheral nerves and cutaneous nerves was done. Reactions and deformities were also noted.

Skin biopsy was done from the margin of the skin lesion and fixed in $10 \%$ formalin. The specimen was processed routinely in the histopathology lab and sections were stained with Haematoxylin and Eosin.

Haematoxylin and Eosin stained sections of the skin biopsies of all the cases of leprosy included in the study were examined for : a) Epidermal atrophy, epithelioid granulomas, number and distribution of lymphocytes, histiocytes \& foam cells. b) Infiltration of nerves, blood vessels and adnexa. c) Grenz zone.

Sections stained with Fite- Faraco stain were examined for lepra bacilli. The following Fite Faraco staining procedure was carried out : 4 micron thick sections were kept in the incubator for 10 to 20 minutes to dewax the sections. The slides were kept in a mixture of xylene and oil (liquid paraffin) in the ratio of (3:2) for 40 minutes. The slides were drained, blotted, dried and rinsed in water for 1 minute, followed by this the sections were covered with carbol fuschin for 40 minutes. The slides were rinsed in water, decolourised with $5 \%$ sulphuric acid for 5 minutes. The slides were washed in water, counter stained with haematoxylin for 3 minutes. Followed by this, slides were washed in water, air dried and mounted with DPX. The stained slides were observed under microscope, mycobacterium appears as pink to red rods in a blue background. Based on Ridley's logarithimic scale, bacterial index (BI) was done. Histopathological findings were graded into tuberculoid (TT), borderline tuberculoid (BT), mid borderline (BB), borderline lepromatous(BL) and lepromatous leprosy (LL) according to Ridley \& Jopling scale. Sections showing scattered non specific lympho-histiocytic infiltration with cellular reaction within the dermal nerve or presence of bacilli in arrector pilorum muscle / dermal nerve were classified as indeterminate leprosy and also included in the study for the purpose of analysis.

Table 1: Histological Types of Leprosy

\begin{tabular}{|l|c|c|}
\hline HISTOLOGICAL TYPE & NUMBER OF CASES & PERCENTAGE \\
\hline TT & 16 & $22.85 \%$ \\
\hline BT & 4 & $5.72 \%$ \\
\hline BB & 4 & $5.72 \%$ \\
\hline BL & 6 & $8.57 \%$ \\
\hline LL & 12 & $17.14 \%$ \\
\hline HISTOID & 4 & $5.72 \%$ \\
\hline IL & 24 & $34.28 \%$ \\
\hline
\end{tabular}

Clinical diagnosis of leprosy cases (as provided by Department of Dermatology, Venerology \& Leprosy) using Ridley \& Jopling scale was correlated with the results of histopathological examination of their respective biopsies.

\section{Results}

During the study period of 2 years from January 2015 to Dec 2016, 70 skin biopsy specimens of clinically diagnosed new untreated cases of leprosy were studied, which included 46 males and 24 females $(\mathrm{M}: \mathrm{F}=1.9: 1)$ aged between 15 to 65 years.

Histopathological examination of the skin biopsies of 70 patients revealed that, the maximum histopathological cases were seen in IL (Indeterminate) type followed by TT and LL (with its variant Histoid type). Maximum number of cases were seen in the polar spectrum ie). TT \& LL (including its variant Histoid) and indeterminate type.

Based on the Ridley \& Jopling logarthmic scale, bacteriological index (BI) was studied on Fite Faraco stained slides. BI observed was 0 (zero) in case of TT and $5+/ 6+$ in cases of LL and its variant Histoid leprosy.

The distribution of 70 cases on clinical leprosy spectrum based on Ridley \& Jopling scale revealed maximum cases in polar spectrum: TT - 28 cases $(40 \%)$, LL and its variant Histoid -18 cases $(14+4)(25.71 \%)$, borderline $(\mathrm{BT}+\mathrm{BB}+\mathrm{BL})-20$ cases $(28.57 \%)$ and the least in IL - 4 cases $(5.72 \%)$. Complete agreement of clinical and histological diagnosis was seen in Histoid Hansen's (variant of LL) (100\%) and IL (100\%) followed by LL $(85.71 \%) \&$ TT $(57.14 \%)$. Least agreement of clinical and histopathological diagnosis was observed in borderline spectrum (BT, BB, BL). Most number of cases which had disparity between clinical and histopathological diagnosis showed histological features of IL (20 cases) due to the absence of granuloma.

In the present study the histopathological characteristics were consistent with the clinical diagnosis in 44 cases out of 70 cases. Complete agreement between clinical diagnosis and histopathology was observed in $62.85 \%$ and disagreement was seen in $37.15 \%$ cases. 
Table 2: Correlation of Hpe Diagnosis and Bacteriological Index ( Fite Faraco Stain).

\begin{tabular}{|l|c|}
\hline HPE DIAGNOSIS & BACTERIOLOGICAL INDEX (BI) \\
\hline TT & 0 \\
\hline BT & $1+$ \\
\hline BB & $2+/ 3+$ \\
\hline BL & $3+/ 4+$ \\
\hline LL & $5+$ \\
\hline HISTOID & $5+/ 6+$ \\
\hline IL & $0 / 1+$ \\
\hline
\end{tabular}

Table 3: Clinico- Histopathological Correlation

\begin{tabular}{|l|c|c|c|c|c|c|c|c|}
\hline \multirow{2}{*}{ CLINICAL DIAGNOSIS } & \multicolumn{9}{|c|}{ HISTOPATHOLOGICAL DIAGNOSIS } & \multicolumn{2}{c|}{ PARITY } \\
\cline { 2 - 9 } & TT & BT & BB & BL & LL & HISTOID & IL & PERCENTAGE \\
\hline TT (28) & 16 & - & - & - & - & - & 12 & $57.14 \%$ \\
\hline BT (8) & - & 2 & 2 & - & - & - & 4 & $25 \%$ \\
\hline BB (4) & - & - & 2 & - & - & - & 2 & $50 \%$ \\
\hline BL (8) & - & 2 & - & 4 & - & - & 2 & $50 \%$ \\
\hline LL (14) & - & - & - & 2 & 12 & - & - & $85.71 \%$ \\
\hline HISTOID(4) & - & - & - & - & - & 4 & - & $100 \%$ \\
\hline IL(4) & - & - & - & - & - & - & 4 & \\
\hline
\end{tabular}

Table.4. Complete Agreement - Clinical Diagnosis \& Histopathology

\begin{tabular}{|l|c|c|c|}
\hline \multicolumn{2}{|c|}{ CLINICAL CASES } & \multicolumn{2}{c|}{ COMPLETE PARITY ( HPE ) } \\
\hline TYPES & NUMBER & NUMBER & PERCENTAGE \\
\hline TT & 28 & 16 & $57.14 \%$ \\
\hline BT & 8 & 2 & $25 \%$ \\
\hline BB & 4 & 2 & $50 \%$ \\
\hline BL & 8 & 4 & $50 \%$ \\
\hline LL & 14 & 12 & $85.71 \%$ \\
\hline HISTOID & 4 & 4 & $100 \%$ \\
\hline IL & 4 & 4 & $100 \%$ \\
\hline TOTAL & $\mathbf{7 0}$ & $\mathbf{4 4}$ & $\mathbf{6 2 . 8 5 \%}$ \\
\hline
\end{tabular}

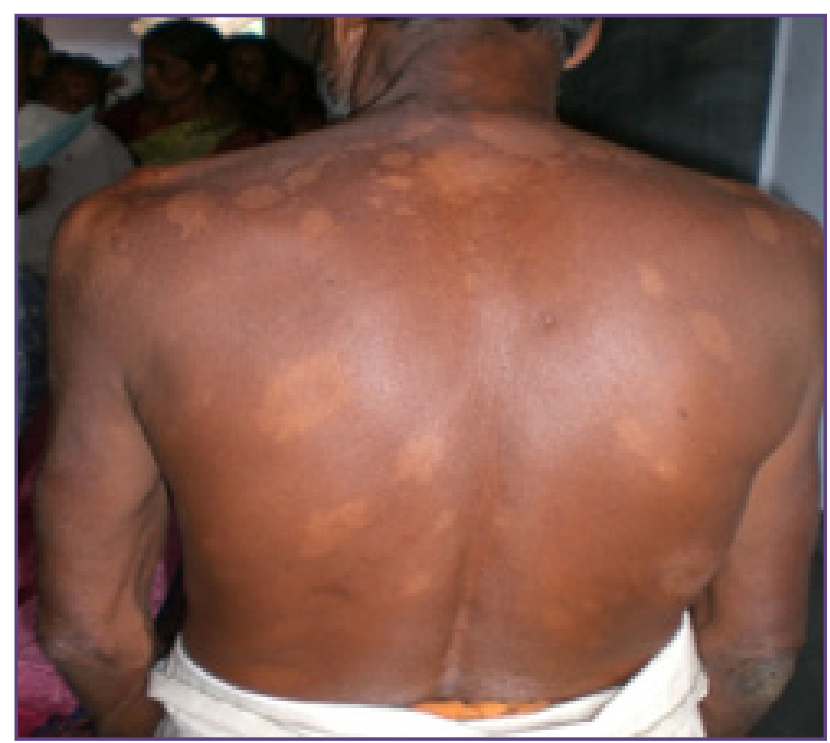

Fig.1: Borderline lepromatous leprosy. Multiple bilateral more or less symmetrical copper coloured shiny anaesthetic patches.

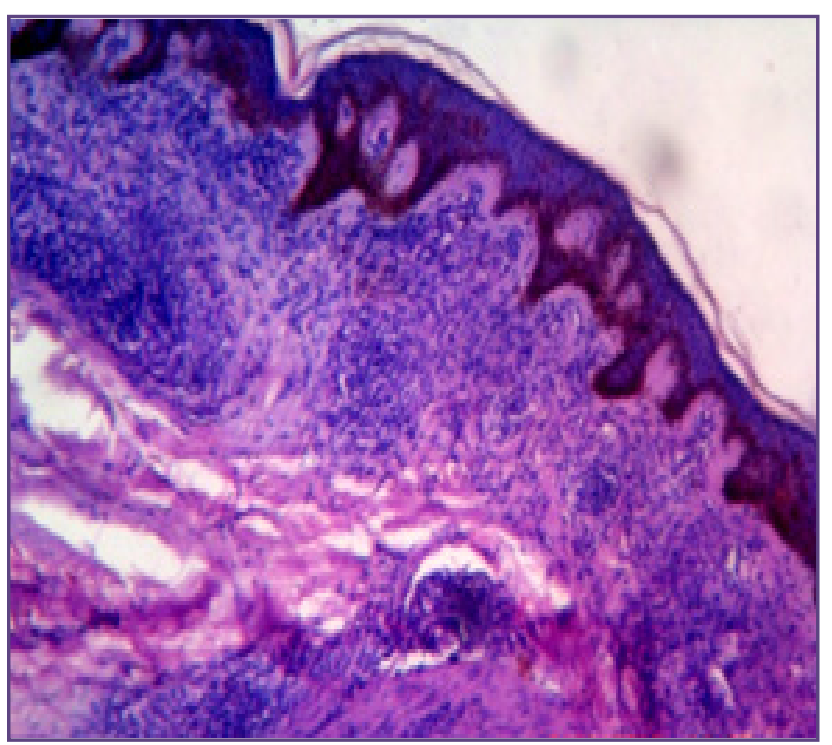

Fig. 2: Borderline lepromatous leprosy : Atrophic epidermis, clear subepidermal grenz zone, collection of macrophages and lymphocytes. 


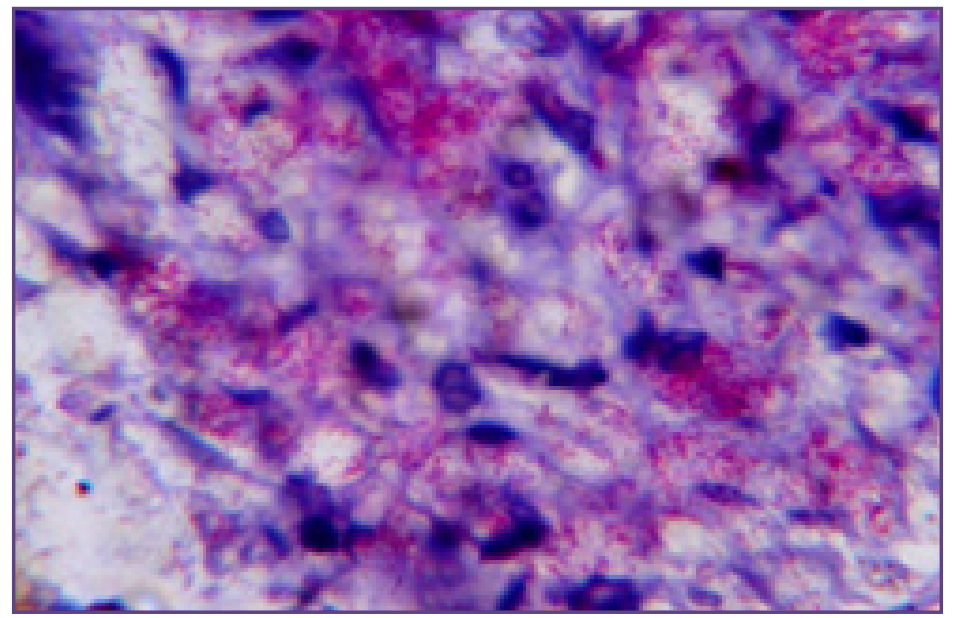

Fig. 3: Borderline lepromatous leprosy. Fite- Faraco stain. Bacterial Index ( BI ) = 4+.

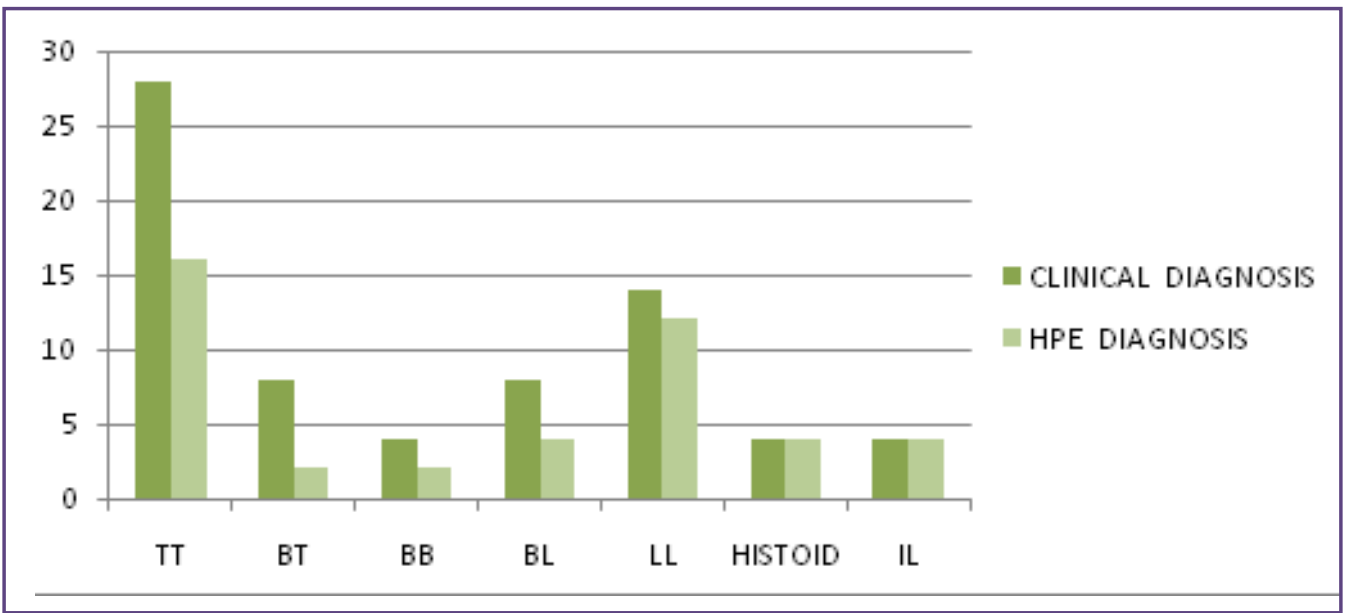

Fig. 4: Clinico - Histopathological correlation of various types of leprosy. Y- axis denotes the number of patients.

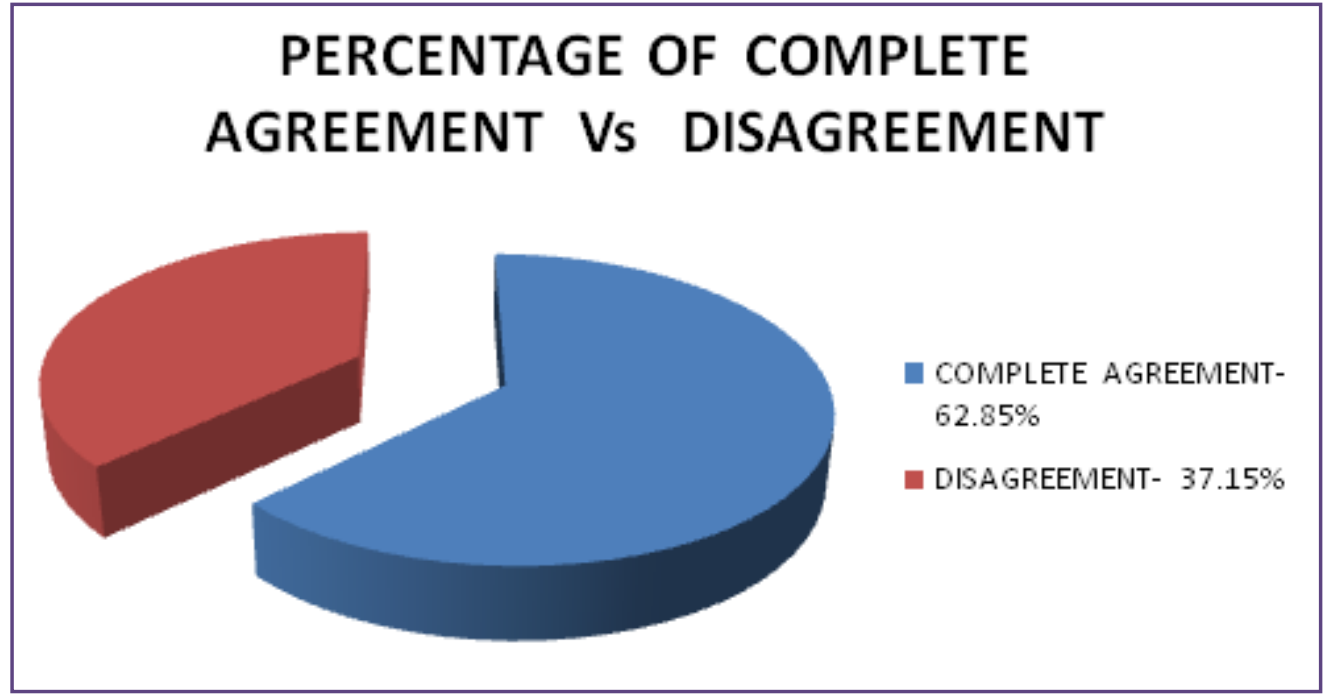

Fig. 5: Percentage of complete agreement of histopathological diagnosis with clinical diagnosis and percentage of disagreement. 


\section{Discussion}

A chronic disease like leprosy needs appropriate classification because of its varied manifestations. The most commonly accepted classification by research workers is that of Ridley \& Jopling ${ }^{4}$ which is primarily based on immunity but has been correlated with clinical, histopathological and bacteriological findings.

Despite having such an accurate classification, there are diversities between the clinical and histopathological features. Clinical spectrum of leprosy in the present study revealed maximum cases in polar spectrum - TT (40\%), followed by LL and its variant Histoid (25.71\%), borderline (ie) BT, BB, BL (28.57\%) and IL (5.72\%). Similar predominance of cases in polar spectrum was observed by Kalyani mitra et $\mathrm{al}^{5}$. In the present study the histopathological characteristics were consistent with the clinical diagnosis in 44 cases out of 70 cases $(62.85 \%)$, similar to the observation made by Nadia et $\mathrm{al}^{6}$. Lepromatous cases (including its variant Histoid) seem to present the least problem in classification. Similar highest percentage of agreement between clinical and histopathological diagnosis of lepromatous leprosy cases was also observed by Shenoi \& Sidappa ${ }^{7}$, Pandey \& Tailor ${ }^{8}$, Bhatia et $\mathrm{al}^{9}$, Kalla et $\mathrm{al}^{10}$ and Shanker Naryan et $\mathrm{al}^{11}$ in their respective studies.

Least agreement was seen in cases of borderline spectrum (BT, BB, BL) in this study, which is in concordance with observations recorded by Shenoi \& Siddappa $^{7}$, Nadkarni \& Rege ${ }^{12}$, Bhatia et $\mathrm{al}^{9}$ and Singhi et $\mathrm{al}^{13}$. Maximum discordance (37.15\%) between clinical and histopathological diagnosis was observed in borderline spectrum cases (BT, BB, BL) of the present study and the same was also noted by Singhi et $\mathrm{al}^{13}$ and Sandeep et $\mathrm{al}^{14}$. Borderline spectrum particularly midborderline leprosy is immunologically the least stable and a variety of clinical lesions of different morphology may be found in the same patient. It is therefore necessary to relate the histological features with the clinical characteristics presented by the particular morphological lesion subjected to biopsy. If this is done carefully, it may be possible to achieve a better clinical correlation with the histological changes.

Tuberculoid and borderline tuberculoid leprosy often overlap clinically, histologically and immunologically but differ only in the degree and the same is true for borderline lepromatous and lepromatous leprosy.

In the present, study, 24 cases (34.28\%) were diagnosed as indeterminate leprosy histologically as against 4 cases $(5.72 \%)$ clinically. Nadkarni \& Rege ${ }^{12}$ and Kalyani mitra et $\mathrm{al}^{5}$ had also diagnosed a sizeable proportion of the cases as indeterminate leprosy histopathologically, who were clinically classified as cases of TT, BT, BB or BL leprosy. Indeterminate leprosy is one which cannot be classified within the Ridley \& Jopling spectrum due to lack of distinguishing features and this happens more often histologically (due to the failure to find a granuloma) than clinically. In the present study, the high percentage of indeterminate leprosy noted histologically in clinical cases of TT, BT \& BB groups could have been due to immunological differences in host responses.

The disparity between clinical and histological observation was anticipated because the parameters used for the histopathological classification are well-defined, precise and also take into account the immunologic response of the tissue, while the clinical classification gives recognition only to the gross appearances of the lesions, which is due to the underlying pathological changes. Moreover, a sizable proportion of leprosy cases (BT, BB, BL) are in a continuously changing immunological spectrum and the histological classification gives a better indication for any recent shift of a case position in the spectrum.

In some early cases, clinical signs and symptoms may precede the presently known characteristic tissue changes, or vice versa'. If a biopsy is taken at an early stage, there is likely to be discordance between the clinical and histopathologic observation. As disparity depends upon the lesion biopsied at the time of study, biopsy from the lesion which is morphologically suggestive of clinical diagnosis, serial biopsies from the same lesion or from paired lesions should be studied for better clinico pathological correlation.

From the present study it was observed that :

- The commonest age group affected by leprosy was 31 - 50 years.

- Males are twice more commonly affected by leprosy than females. $(\mathrm{M}: \mathrm{F}=1.9: 1)$.

- The most commonest clinically diagnosed spectrum was tuberculoid leprosy (TT).

- The commonest histopathologically diagnosed spectrum was IL, followed by TT \& LL with its variant histoid leprosy.

- It was observed that there was complete agreement between clinical diagnosis and histopathological diagnosis in $62.85 \%$ cases.

- Disagreement between histopathological diagnosis and clinical diagnosis was observed in $37.15 \%$ cases. 
- If there is discrepancy between clinical and histopathological classification, both the findings should be reviewed by independent experienced observers.

- In case of confirmed discrepancy, the more advanced findings (ie. towards the lepromatous pole) should be given greater weightage and the case is to be classified and treated accordingly.

- In all cases of leprosy, in order to type the exact spectrum of disease for appropriate treatment, skin biopsy of the lesions with histopathological examination followed by special stain (Fite -Faraco) is recommended.

\section{Conclusion}

In clinical practice a case of leprosy is to be classified as per clinical criteria. Skin biopsy has to be taken from the most active site of the lesion. This will help in the confirmation of diagnosis and classification. If there is discrepancy between clinical and histopathological classification both the findings should be reviewed by independent experienced observers. In case of confirmed discrepancy the more advanced findings (ie. towards the lepromatous pole) should be given greater weightage and the case is to be classified and treated accordingly. This will prevent inadequate treatment of a particular case.

\section{References}

1. Jopling WH, McDougall. Definition, Epidemiology and World Distribution Hand book of Leprosy, Fifth edition. CBS Publishers and Distributors 1996: 1.

2. National Leprosy Eradication Programme - Annual report for the year $2015-2016$.
3. WHO - Fact sheet on Leprosy : Status of the disease in 2015.

4. Ridley DS, Jopling WH. Classification of leprosy accroding to immunity: a five- group ystem. Int J Lepr 1966; 34: 255.

5. Mitra K, Biswas $\mathrm{S}$ et al. Correlation between clinical and histopathological criteria for the classification of leprosy. Indian J Dermatol 2001; 46 (3) :135- 137.

6. Nadia S, Rashmi J, Sohaib A, Rawat SDS, Selvi Thamarai N, Meena H. Clinicopathological correlation of Leprosy : A 4 year's retrospective study from a tertiary referral centre in North India. Int J Med Res Health Sci. 2015;4(2):350-354.

7. Shenoi SD, Siddappa K. Correlation of clinical and histopathologic features in untreated macular lesions of leprosy - a study of 100 cases. Ind J Lepr 1988; 60 : 202 - 06.

8. Pandey AN, Tailor HJ. Clinicohistopathological correlation of leprosy. Ind J Dermatol Venereol Lep 2008; 74 : 74- 76.

9. Bhatia AS, Katoch $\mathrm{K}$ et al. Clinical and histopathological correlation in the classification of leprosy. Ind J Lepr 1993 ; 61 : 433- 438.

10. Kalla G, Salodkar A, Kachhawwa D. Clinical and histopathological correlation in leprosy. Ind J Lepr 2000 ; $68: 184-185$.

11. Shanker Narayan NP, Ramu G et al. Correlation of clinical, histological and immunological features across the leprosy spectrum. Ind J Lepr 2001 ; 73 :329- 42.

12. Nadkarni NS, Rege VL. Significance of histopathological classification in leprosy. Ind J Lepr 1999 ; 7 : 325- 32.

13. Singhi MK, Kachhawa D, Ghiya BC. A retrospective study of clinic- histological correlation in leprosy. Ind J Pathol Microbiol 2003 ; 46 : 47- 48.

14. Sandeep M, Shamanur Murugesh. A Study of ClinicPathological Concordance in Leprosy Patients in the PostElimination Era. International Journal of Science and Research. 2016;5(2):1304-1306.

*Corresponding author:

DrVennila Muniswamy, Dept. of Pathology, Government Vellore Medical College and Hospital, Adukamparai, Vellore, Tamil Nadu 632011, India. Phone: +918939958993

Date of Submission : 06.04.2017

Date of Acceptance : 26.04.2017

Financial or other Competing Interests: None.

Date of Publication : 31.08.2017 